\title{
Fault Detection Scheme for a Road Vehicle With Four Independent Single-Wheel Electric Motors and Steer-By-Wire System
}

\author{
Bruno dos Santos, António Lopes, Rui Esteves Araújo \\ INESC TEC, Faculty of Engineering, University of Porto, PORTUGAL
}

\begin{abstract}
Unknown input observers (UIO) can be used in the model-based fault diagnosis (FD) system to reduce or eliminate the effect of unknown disturbances present on the process and used to create a set of residuals that are decoupled and sensitive to faults. In this work, a new FD scheme of the In-Wheel motors electric vehicle (IWM-EV) with active front steering was carried out, as well as the design of the fault isolation banks of UIOs. These banks are used to generate residuals that are robust againts to noise and are sensitive to only one fault. This way the faults in the steering or in-wheel actuator are detected and isolated with a higher

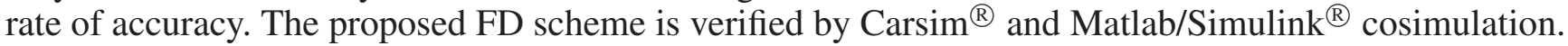

\section{INTRODUCTION}

The road map on road transport published by ERTRAC, EPOSS and SMARTGRIDS in 2010 had the expectation that the adaptation of conventional vehicles to electric power train was the first milestone (Ertrac, EPoSS, \& SmartGrids 2012). At the present stage, most compact electric vehicle (EV) are based on today's drive train platforms with one central electric motor. However the potential of novel power drive concepts is not completely achieved with these first generation vehicles. Multiple electric motors mounted close to the wheels or even in the wheel opens the development of new compact vehicle drive train architectures.

System redundancy could be explored in order to achieve high safety and robustness requirements. The redundancy available in multi-motor electric vehicles should be exploited, in the event of an actuator failure, to redistribute the control effort among the remaining working actuators such that stability is retained and recover to a safe state. Also (Zhang, Cocquempot, Jiang, \& Yang 2013) and (Rongrong \& Junmin 2011) points the importance of using fault detection and identification (FDI) scheme with fault tolerant control (FTC) systems in order to actively reconfigure the system in order to mitigate the faults effects. Several works have been presented related to the detection of fault in electric vehicles. In (Ho \& Ossmann 2014) it is used structured residuals in order to detect and isolate fault in sensors and actuators. In this work the linear track-model with side slip state is extended to the new type of powertrain is used.

In this work, we are concerned with the fault diag- nosis system for an IWM-EV with active front steering. The selected powertrain is equipped with four independent electric drives, which can individually generate traction and braking forces at the tires. The steering system is assumed to be a steer-by-wire (SBW) system which is able to control the front wheel steering angle independently of the drivers input with the steering wheel. Due to the high complexity associated with this new class of powertrains, the probability of malfunctions in components (sensors, actuators) is increasing. In this context, it is crucial to develop new algorithms for FD of vehicle components and increasing vehicle safety using fault diagnosis systems.

The main objective of this work is to present a new FD for the vehicle motion controller. To this aim, we propose a structure based on three processing blocks (see figure 1). The first processing block is related to wheel fault detection: a wheel force observer was employed to estimate the difference between the expected forces and the ones applied in the vehicle. In the second block, we employ the inverse model of the yaw rate in order to reconstruct the steering input. For that we use an observer to estimate the lateral velocity of the vehicle. The last block is composed by a set of bank of observers based on the vehicle dynamics in the yaw rate and longitudinal velocity.

The paper is organized as follows. In section 2 the vehicle model is presented. The fault detection scheme and some details are presented in section 3. In section 4 the supervision algorithm, that isolates the fault based on the residual is explained. The FD simulation is presented in section 5 with some discussion about the results. In the final section some conclusions and insights for the future work are discussed. 


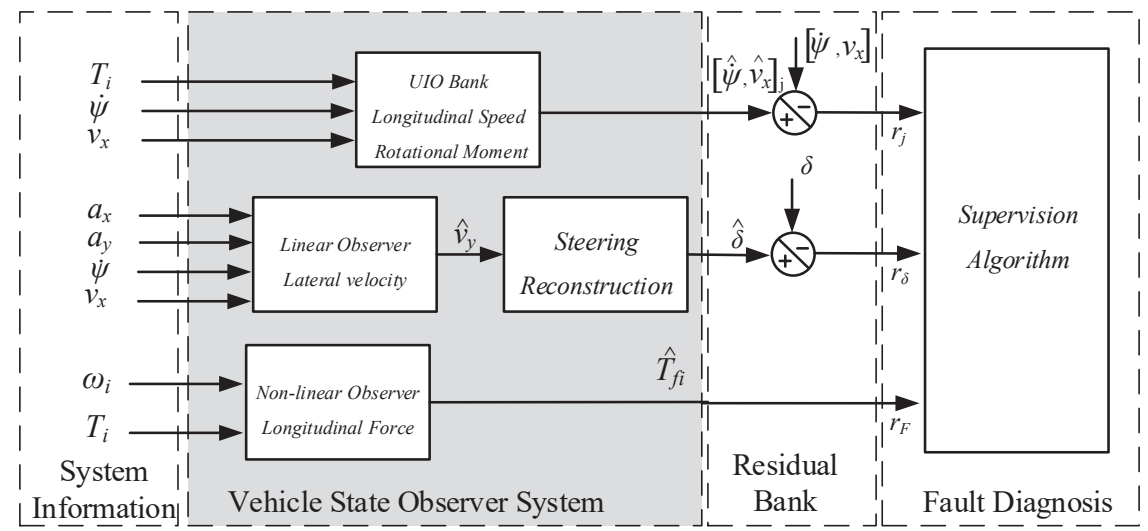

Figure 1: Diagram of the fault diagnosis algorithm developed

\section{VEHICLE MODEL}

In this section the vehicle model used in order to design the FDI scheme is explained bellow. The rotational dynamic of the wheel is presented as (Savaresi \& Tanelli 2010):

$$
\begin{array}{r}
I_{w} \dot{\omega}_{i}=-R \cdot\left(F_{x i}+F_{r i}\right)+T_{i} \\
i \in\{F L, F R, R L, R R\}
\end{array}
$$

where $T_{i}$ are the torque inputs applied in each individual wheel and $I_{w}$ is the wheel moment of inertia and $R$ is the wheel radius. The $F_{r i}$ is the wheel rolling forces. Finally, the forces $F_{x i}$ arise from the tire/road interaction. The linear force is represented by the following model:

$F_{x i}=N_{i}\left(\frac{R \omega_{i}-v_{\omega i}}{v_{\omega i}}\right)$

where $N_{i}$ is the longitudinal stiffness coefficient, $v_{\omega_{i}}$ and $\omega_{i}$ are the linear and angular wheel speed, for $i \in$ $\{F L, F R, R L, R R\}$.

The total lateral force $F_{y}$ of the system is expressed by (3) and it is assumed that there is no difference between the lateral forces applied in the left and right tires.

$F_{y}=2 F_{y f}+2 F_{y r}$

Where $F_{y f}$ and $F_{y r}$ represents the lateral force present in the front and rear wheels respectively. In the present formulation it will be considered that the lateral forces are defined as a linear model expressed by:

$F_{y f}=C_{f}\left(\delta-\frac{v_{y}+l_{r} \dot{\psi}}{v_{x}}\right), \quad F_{y r}=C_{r}\left(\frac{l_{f} \dot{\psi}-v_{y}}{v_{x}}\right)$

The vehicle motion can be expressed by the Newton - Euler equations which describes the lateral and longitudinal dynamics of the CoG of a rigid body
(Jazar 2008). The presented planar model is based on the rotational, and translational motion of the vehicle, neglecting the vertical dynamic of the suspension system. The proposed model is based on the two-track vehicle model and is defined as:

$$
\begin{cases}\dot{v}_{x} & =v_{y} \dot{\psi}+\frac{1}{m}\left(\sum_{y}\left(F_{x i}+F_{r i}\right)+F_{w i n d}\right) \\ \dot{v}_{y} & =-v_{x} \dot{\psi}+\frac{F_{y f}+F_{y r}}{m} \\ I_{z} \ddot{\psi} & =l_{f} F_{y f}-l_{r} F_{y r} \\ & +0.5 \cdot l_{s}\left(F_{x F L}+F_{x R L}-F_{x F R}-F_{x R R}\right)\end{cases}
$$

The constant of $l_{f}, l_{r}$ and $l_{s}$ express the distance of the front and rear axis to the center of gravity of the system and the distance between the wheels of the vehicle. The vehicle mass is symbolized by $m$ and the rotational moment of inertia of the vehicle is defined as $I_{z}$. The state variables of the system are the longitudinal and lateral velocity of the vehicle, $v_{x}$ and $v_{y}$ respectively, and the yaw rate $\dot{\psi}$. The force $F_{\text {wind }}$ is the aero-drag force. Also the longitudinal wheel force $F_{x i}$ is the one defined in (2).

The nonlinear system is linearised around the equilibrium point $\mathbf{x}_{0}=\left[\begin{array}{lll}V & 0 & 0\end{array}\right]^{T}$. Also the wheel angular acceleration is $\dot{\omega}_{i}=0$ and the rolling forces are omitted. In this conditions the fault free linear system is obtained:

$\left\{\begin{array}{l}\dot{\mathbf{x}}=A \mathbf{x}+B \mathbf{u} \\ \mathbf{y}=C \mathbf{x}\end{array}\right.$

where:

$\mathbf{x}=\left[\begin{array}{c}v_{x} \\ v_{y} \\ \dot{\psi}\end{array}\right], \quad \mathbf{y}=\left[\begin{array}{c}v_{x} \\ \dot{\psi}\end{array}\right], \quad \mathbf{u}=\left[\begin{array}{c}F_{x l} \\ F_{x r} \\ \delta\end{array}\right]$

$A=\left[\begin{array}{ccc}-2 K_{w} V & 0 & 0 \\ 0 & \frac{-2\left(C_{f}+C_{r}\right)}{m V} & \frac{-2\left(l_{f} C_{f}-l_{r} C_{r}\right)-m V^{2}}{m V} \\ 0 & \frac{-2\left(l_{f} C_{f}-l_{r} C_{r}\right)}{I_{z} V} & \frac{-2\left(l_{f}^{2} C_{f}+l_{r}^{2} C_{r}\right)}{I_{z} V}\end{array}\right]$,

$B=\left[\begin{array}{ccc}\frac{1}{m} & \frac{1}{m} & 0 \\ 0 & 0 & \frac{2 C_{f}}{m} \\ \frac{l_{s}}{2 I_{z}} & \frac{-l_{s}}{2 I_{z}} & \frac{2\left(l_{f}+l_{r}\right) C_{f}}{I_{z}}\end{array}\right], C=\left[\begin{array}{lll}1 & 0 & 0 \\ 0 & 0 & 1\end{array}\right]$. 


\subsection{Fault Model}

Actuators faults can be represented as additive faults (Isermann 2006). When a fault occurs, the control input variable presents a faulty component which is modelled by the equation (7). In this situation, the fault free model control input $\mathbf{u}$ is now defined by $\mathbf{u}_{f}$ which is the new control variable when the faults are considered. In this conditions, it is considered the fault presents in each individual wheel actuator $\left(f_{F L}, f_{F R}, f_{R L}, f_{R R}\right)$ and in the steering wheel $\left(f_{\delta}\right)$ in the fault vector $\mathbf{f}$. The steering fault is exclusive to the front axes. Faults on the left and right wheel cannot be isolated using only the linear model. The isolation between front and rear wheel is made by the wheel force observer.

$$
\begin{aligned}
\mathbf{u}_{f} & =\mathbf{u}\left(I_{3}-\left[\begin{array}{lllll}
1 & 0 & 1 & 0 & 0 \\
0 & 1 & 0 & 1 & 0 \\
0 & 0 & 0 & 0 & 1
\end{array}\right] \mathbf{f}\right) \\
\mathbf{f} & =\left[\begin{array}{lllll}
f_{F L} & f_{F R} & f_{R L} & f_{R R} & f_{\delta}
\end{array}\right]^{T}
\end{aligned}
$$

\section{FAULT DIAGNOSIS STRUCTURE}

The proposed fault detection and diagnosis scheme is presented in figure 1. This solution is composed by three main elements: the system observers, defined by multiple unknown input observers that estimate the fault free state, the residual bank, where the observed variables are compared with the actual system information, and the fault diagnosis algorithm. The system information is obtained by means of sensor measurements provided by Inertial Measurement Unit (IMU) sensors as well as the expected actuator inputs provided by the control system.

\subsection{Wheel Force Observer}

In order to reconstruct the longitudinal force in each individual wheel, it is proposed a wheel force observer based on sliding mode techniques. The following observer is defined for each wheel $i \in$ $\{F L, F R, R L, R R\}$ and is based on the rotational dynamic presented in (1):

$\dot{\hat{\omega}}_{i}=b_{i} T_{i}-\frac{R \hat{F}_{x i}}{I_{\omega}}+l_{i} e_{i}+v_{i}$,

where $b_{i}=1 / I_{w}, l_{i}$ is the linear observer gain, $\hat{F}_{x i}$ is the linear force estimation using equation (2) and $v_{i}$ is the following discontinuous term:

$v_{i}=\left(\rho_{i}+\rho_{0 i}\right) \operatorname{sign}\left(e_{i}\right)$
Let's assume a quadratic Lyapunov function, $V_{i}=$ $e_{i}^{2} / 2$, where $e_{i}=\omega_{i}-\hat{\omega}_{i}$. The derivative of the proposed function would be defined as:

$\dot{V}=-l_{i} e_{i}^{2}+e_{i}\left(\frac{-R F_{x i}+T_{f i}}{I_{w}}+\frac{R \hat{F}_{x i}}{I_{\omega}}-v_{i}\right)$

Considering $v_{i}$ as (9) and assuming $\left|\rho_{0 i}\right|>$ $R\left|F_{x i}-\hat{F}_{x i}\right|^{\max }+T_{f i} / I_{w}$, where $T_{f i}$ is the faulty component of $\boldsymbol{T}_{\boldsymbol{i}}$. The result (10) is reduced to:

$\dot{V} \leq-l_{i} e_{i}^{2}-\rho_{i}\left\|e_{i}\right\|<0$

Ensuring asymptotic stability for the proposed observer.

In order to estimate the wheel fault, it is first assumed that both the error and his derivative are zero. With these assumptions the error expression (with $\rho_{i} \gg l_{i}$ and $F_{x i}-\hat{F}_{x i} \approx 0$ ) is:

$$
\dot{e}_{i}=0 \Leftrightarrow \frac{R F_{x i}-T_{f i}}{I_{w}}=\frac{R \hat{F}_{x i}}{I_{w}}-l_{i} e_{i}-v_{i} \Leftrightarrow
$$

$\hat{T}_{f i} \approx I_{\omega} v_{i}$

\subsection{Steering Reconstruction}

The proposed steering reconstruction is based on the yaw rate model defined previously in (5) where the lateral forces are modelled by (4).

Almost all the variable are available. In order to simplify the calculation the yaw-rate derivative is set to be close zero $\ddot{\psi} \approx 0$. Nonetheless, it is assumed that the information of the lateral velocity is not available for measurement, which hinders the estimation of the steering variable. In order to overcome this difficulty, a linear observer is designed in order to closely track the lateral velocity of the system.

The state space system of the longitudinal/lateral motion is used from the system of equation (6), the observer can be defined as:

$$
\left\{\begin{array}{l}
{\left[\begin{array}{l}
\hat{\dot{v}}_{x} \\
\dot{\hat{v}}_{y}
\end{array}\right]=\left[\begin{array}{cc}
0 & \dot{\psi} \\
-\dot{\psi} & 0
\end{array}\right]\left[\begin{array}{l}
\hat{v}_{x} \\
\hat{v}_{y}
\end{array}\right]+\left[\begin{array}{l}
a_{x} \\
a_{y}
\end{array}\right]+L \mathbf{e}_{y}} \\
\hat{\mathbf{y}}=\left[\begin{array}{ll}
1 & 0
\end{array}\right]\left[\begin{array}{l}
\hat{v}_{x} \\
\hat{v}_{y}
\end{array}\right]
\end{array}\right.
$$

where the longitudinal/ lateral wheel forces are replaced by the acceleration measured by the sensors. The observer gain matrix is represented by $L$ which is defined so that the resulting observer error $\mathbf{e}_{y}=$ $(\hat{\mathbf{y}}-\mathbf{y}) \rightarrow 0$ when $t \rightarrow \infty$. The observation stability is obtained by defining the following gain matrix (for more information refer to (Ungoren, Peng, \& Tseng 2002)):

$L=\left[\begin{array}{ll}2 a \mid \dot{\boldsymbol{\psi}} & \left(a^{2}-1\right) \dot{\boldsymbol{\psi}}\end{array}\right]^{T}$ 
where $a$ is a positive scalar. However, the system observability presented in (12) is lost when the yaw rate variable becomes zero, in order to overcame this shortage, the fault detection is only valid for $\dot{\psi} \neq 0$.

\subsection{UIO Bank with fault isolation}

The structure of the UIO bank is presented in the figure 2. As already mentioned, the measurements obtained from the sensors are fault free and the fault detection scheme is defined for actuators faults only. The UIO bank consists of two different observers (figure 2) that aim to decouple actuators faults obtaining a clear estimation that will dissociate the system faults (Chen \& Patton 1999). The model for each UIO is based on the linear model defined in equation (6). The control input is replaced by the fault model presented in equation (7). The resulting system is described by:

$$
\left\{\begin{array}{l}
\dot{\mathbf{x}}_{j}=A \mathbf{x}_{j}+B_{j} \mathbf{u}_{j}+D_{j} \mathbf{d}_{j}+B_{f j} \mathbf{f}_{j} \\
\mathbf{y}_{j}=C \mathbf{x}_{j}
\end{array}\right.
$$

where $B_{j} \in \Re^{n \times(m-1)}$ is the system input matrix $B$, without the column regarding the unknown input $j$; $b_{j} \in \Re^{n \times 1}$ refers to the column of the matrix $B$ that was isolated from $B_{j} ; D_{j}=\left[b_{j}\right]$ is the new perturbation distribution matrix. The fault distribution $B_{f j} \in \Re^{n \times(m-1)}$ is equal to $B_{j}$.

The UIO observer based on residual generation that detect and isolate faults has a structure as described in the following system of expressions:

$$
\left\{\begin{array}{l}
\dot{\mathbf{z}}_{j}=F_{j} \mathbf{z}_{j}+T_{j} B_{j} \mathbf{u}_{j}+K_{j} \mathbf{y} \\
\hat{\mathbf{x}}_{j}=\mathbf{z}_{j}+H_{j} \mathbf{y} \\
\hat{\mathbf{y}}_{j}=C \hat{\mathbf{x}}_{j} \\
\mathbf{r}_{j}=V_{j}\left(\mathbf{y}-\hat{\mathbf{y}}_{j}\right)
\end{array}, \quad \text { for } j \in\{1,2\}\right.
$$

where $\hat{\mathbf{x}}_{j} \in \Re^{n}$ corresponds to the estimated state vectors and $\mathbf{z}_{j} \in \Re^{n}$ corresponds to the observer state and $F_{j} \in \Re^{n \times n}, T_{j} \in \Re^{n \times n}, K_{j} \in \Re^{n \times p}, H_{j}, \in \Re^{n \times p}$ are the matrix needed to build the observer with unknown input decoupling. The matrix $V_{j}$ is a gain factor in order to obtain the residual.

The residual for the group of actuator $j$ has only one side of the longitudinal traction as presented in figure 2 . If a fault occurs in the group $j$, The detection

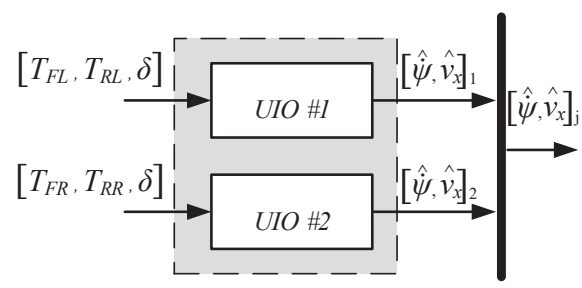

Figure 2: UIO bank observer logic is as following:

$\left\{\begin{array}{l}\left\|r_{j}\right\|<T h_{j} \\ \left\|r_{b}\right\|>T h_{j}\end{array}, \quad\right.$ for $b \in\{1,2\} \backslash j$

where $T h_{j}$ is the threshold value for each actuator.

\section{UIO Design}

The state estimation error is defined by: $\left(\mathbf{e}_{j}=\mathbf{x}_{j}-\right.$ $\hat{\mathbf{x}}_{j}$ ), it follows that $\mathbf{e}_{j}=T_{j} \mathbf{x}_{j}-\mathbf{z}_{j}$, where $T_{j}=I-$ $H_{j} C$. Using the equations (15) and (14), the time derivative of the error is given by (For the sake of simplicity, the index $j$ is omitted):

$$
\begin{aligned}
\dot{\mathbf{e}}= & F \mathbf{e}+(T B-G) \mathbf{u}+(T D) \mathbf{d}+ \\
& +\left(T B_{f}\right) \mathbf{f}+(T A-K C+F H C-F) \mathbf{x}
\end{aligned}
$$

In order to design the UIO, is necessary to met the following conditions:

$$
\text { (C1) } G=T B(\text { cancel } \mathbf{u}) ; \quad \text { (C2) } T D=0 \text { (cancel d) }
$$

(C3) $F=T A-L C$ ( $F$ is "Hurwitz"); (C4) $K=L-F H$

$$
\text { (C5) } \operatorname{rank}\left(T B_{f}\right)=\operatorname{rank}\left(B_{f}\right) \quad(\mathrm{C} 6) T=I-H C
$$

If the conditions are respected the state estimation error becomes $\left(\dot{\mathbf{e}}=F \mathbf{e}+T B_{f} \mathbf{f}\right)$ and the residual corresponds to $\mathbf{r}=V C \mathbf{e}$.

In order to cancel the perturbation it is necessary to first check if $\operatorname{rank}(C D)=\operatorname{rank}(D)$ in order to guarantee a solution for the system $H C D=D$ (from the condition (C2) and (C6)). One generalized solution for $H$ can be expressed by (Darouach, Zasadzinski, \& Xu 1994):

$H=D(C D)^{+}+H_{0}\left(I-(C D)(C D)^{+}\right)$

where $(X)^{+}$is the generalized inverse matrix given by $(X)^{+}=\left((X)^{T}(X)^{-1}\right)(X)^{T}$, since $X$ is full rank. $H_{0}$ is an arbitrary matrix of appropriate dimensions. This matrix give some freedom on the design. The eigenvalues of $F$ can be arbitrary located by choosing a suitable matrix $L$, only if the pair $(T A, C)$ is observable. If this condition can not be applied it is possible to found a $K_{1}$ that the observer is asymptotically stable if and only if $(T A, C)$ are detectable.

\section{SUPERVISION ALGORITHM}

Based on the residuals created, it is possible to isolate and identify faults in the actuators (steering and wheel actuators) using a residual table. The table identifies a possible fault using the several residual signatures.

The response to fault situation in residual $r_{1}$ and $r_{2}$ can be obtained by using the error expression from equation (15). Taking in account the observation error $\dot{\mathbf{e}}=F \mathbf{e}+T B_{f} \mathbf{f}$ and the residual $\mathbf{r}=V C \mathbf{e}$ it is possible to predict the residual behaviour for additive fault. The wheel observer gives the fault signature for 


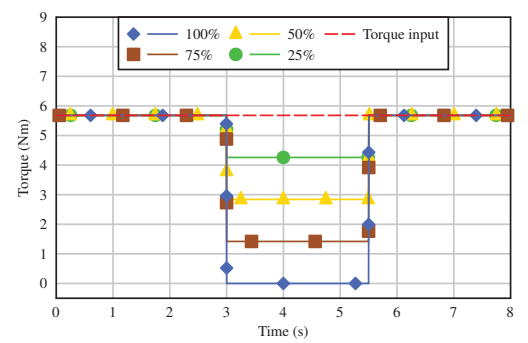

a - Torque input and faults

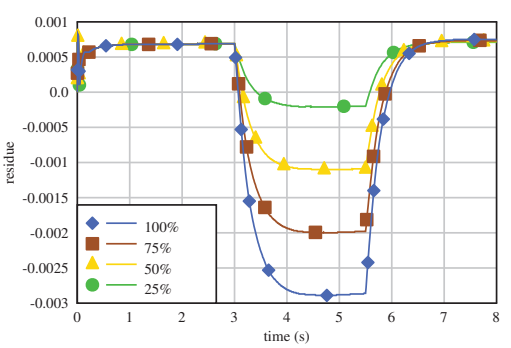

d - Residual $r_{1} \dot{\psi}$

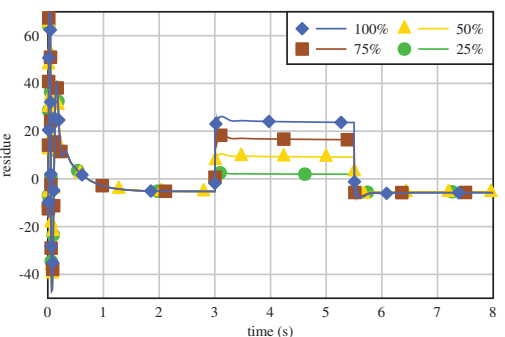

b - Residual $r_{F_{F} R}$

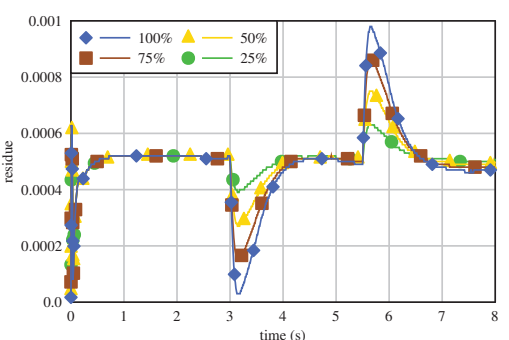

e - Residual $r_{2} v_{x}$

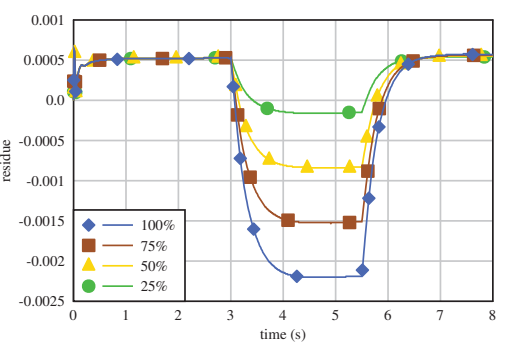

c - Residual $r_{1} v_{x}$

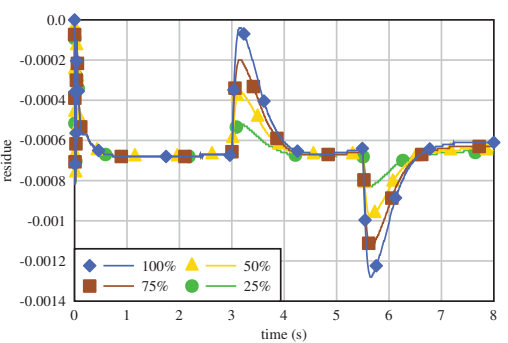

f - Residual $r_{2} \dot{\psi}$

Figure 3: Simulation results for the motor fault test;

each individual wheel and the steering reconstruction provides the information of steering alteration.

The detection is obtained by comparing the residuals with appropriate threshold and its sign is also used. The table 1 shows the possible patterns for the residuals.

\section{SIMULATION RESULTS}

To illustrate the fault detection algorithm scheme performance a computer simulation was carried out. The vehicle model was emulated through the tool Carsim ${ }^{\circledR}$ in co-simulation with Matlab/ Simulink ${ }^{\circledR}$ software where the proposed solution was implemented. In order to demonstrate the capacity to detect faults in actuators two fault scenarios are defined: Firstly without steering input $(\delta=0)$ and constant acceleration the fault is injected in the front right wheel actuator. Secondly, a J-turn maneuver with steer fault is considered. In both case the torque in each wheel is constant and set to $5.6 \mathrm{Nm}$. The vehicle parameters are presented in table 2 . In the simulation gaussian white noise $\left(\sigma^{2}=10^{-5}\right)$ was added to all measured

Table 1: Residual from the several actuator faults; 0 - residual is insensitive against the respective threshold, + residual is sensitive and positive, - residual is sensitive and negative.

Faults in actuators

\begin{tabular}{|c|c|c|c|c|c|c|}
\hline \multirow{3}{*}{$r_{1}$} & & $f_{\delta}$ & $f_{F L}$ & $f_{F R}$ & $f_{R L}$ & $f_{R R}$ \\
\hline & $v_{x}$ & - & 0 & - & 0 & - \\
\hline & $\dot{\psi}$ & - & 0 & - & 0 & + \\
\hline \multirow{2}{*}{$r_{2}$} & $v_{x}$ & + & - & 0 & - & 0 \\
\hline & $\dot{\psi}$ & - & - & 0 & + & 0 \\
\hline \multicolumn{2}{|c|}{$r_{\delta}$} & + & 0 & 0 & 0 & 0 \\
\hline \multirow{4}{*}{$r_{F_{i}}$} & $F L$ & 0 & + & 0 & 0 & 0 \\
\hline & $F R$ & 0 & 0 & + & 0 & 0 \\
\hline & $R L$ & 0 & 0 & 0 & + & 0 \\
\hline & $R R$ & 0 & 0 & 0 & 0 & + \\
\hline
\end{tabular}

signals. The signals are filtered by a low pass filter.

In the first simulation, an abrupt fault is injected in the front right wheel. The result is presented in figure 3 . At $t=3 s$ the torque goes from the initial value to the faulty value. After $2.5 \mathrm{~s}$ the wheel torque goes back to the initial value. In this test several fault amplitude have been tested (figure 3.a). The results present a good detection for faults above $f_{F R}=0.25$. Both residual $r_{1}$ and $r_{2}$ have an offset in the fault free operation. This phenomenon is due to the observer structure $\left(H_{1}\right.$ matrix $)$ and by the unavoidable modelling errors. In other words, the fault diagnosis algorithm uses models which do not fully agree with the real vehicle due to model uncertainties. The residuals react as specified by their respective signatures. The residual $r_{1}$ (figure 3.c and figure 3.d) respond has expected on table 1 . The residual $r_{2}$ (figure 3.e and figure 3.f) is the one that isolates the left torque faults and is insensitive to the right wheel. However, at the fault instant, a perturbation occurs, due to the abrupt change on the input, which will be ignored by the fault evaluation mechanism as the value goes to zero. From the wheel actuator fault it is possible to see that the residual have the same behaviour has the set in signature table.

In the second scenario, a step fault is injected in the front steering axis. The result are presented in figure 4. At $t=3 \mathrm{~s}$ the steering goes from the initial value to the faulty value. In this test several fault amplitude have been tested (figure 4.a). The residual can detect, even for small fault values the steering faults. Both residual $r_{1}$ and $r_{2}$ (figure 4.(c-f)) have a response in the steering change in the fault free operation. This is due to the yaw rate dynamics of the model. Although, after some time, the false fault signal goes to zero. The residuals react as specified in the table 1 by their respective signatures in the presence of fault. As expected, the residuals response for $r_{1}$ and $r_{2}$, associated with the steering fault is more significant than 


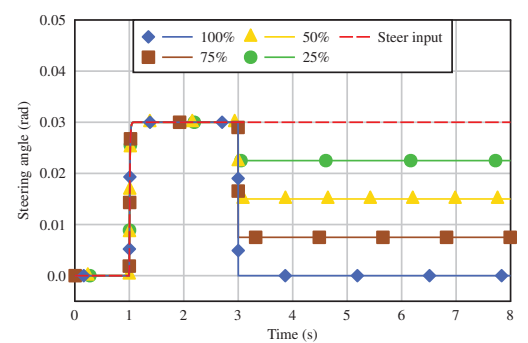

a - Steering input and faults

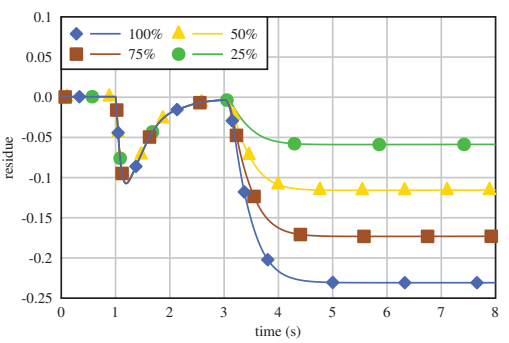

d - Residual $r_{1} \dot{\psi}$

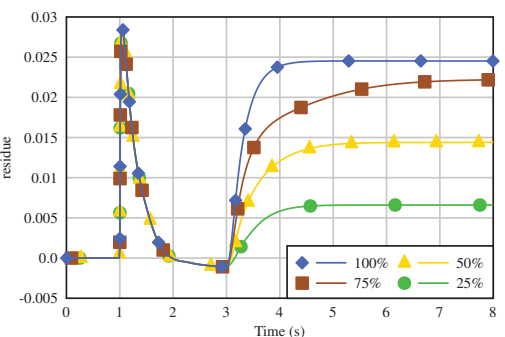

b - Residual $r_{\delta}$

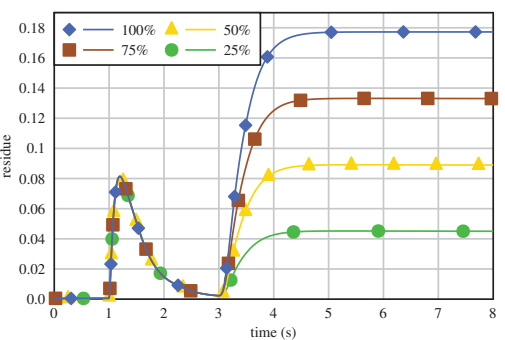

e - Residual $r_{2} v_{x}$

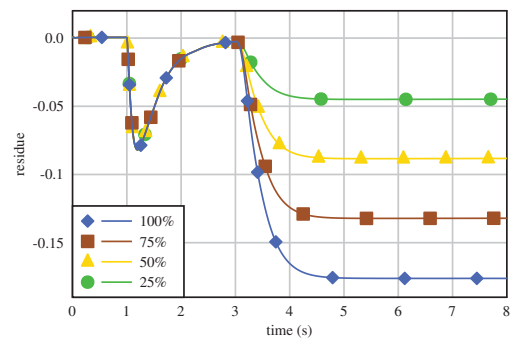

c - Residual $r_{1} v_{x}$

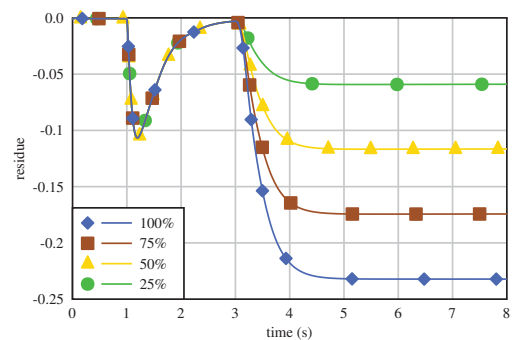

f - Residual $r_{2} \dot{\psi}$

Figure 4: Simulation results for the steer fault test;

the wheel actuator case.

\section{CONCLUSION AND FUTURE WORK}

In this paper, a scheme for detection and isolation of actuator faults in an over-actuated EV was presented. The proposed approach is based on using UIO for calculating a set of residuals. The algorithm was combined with a vehicle state observer consisting of a nonlinear wheel force observer, a linear observer for lateral velocity estimation with steering reconstruction and a set of UIO for longitudinal speed and yaw rate estimations. The observer designs for linearised system have been considered regarding its application to fault diagnosis algorithm. Existence and convergence conditions for the observers have been established. The proposed technical solution allows to decoupling residuals from unknown inputs while keeping sensitivity to faults. The results obtained are promising and motivates further research on improving the proposed approach. The first step could be conducted to reduce sensitivity to the steering input. The fault detection extension in order to detect fault in sensors is the second step. Also, the use of fault detection scheme in order to reconfigure the control system in case of fault is scheduled as future work.

\section{ACKNOWLEDGEMENT}

This work is financed by the ERDF European Regional Development Fund through the Operational Programme for Competitiveness and Internationalisation - COMPETE 2020 Programme within project POCI-01-0145-FEDER-006961, and by National Funds through the Portuguese funding agency, FCT - Fundação para a Ciência e a Tecnologia as part of project UID/EEA/50014/2013 through the scholarship SFRH/BD/90418/2012.

\section{REFERENCES}

Chen, J. \& R. J. Patton (1999). Robust model-based fault diagnosis for dynamic systems. Springer.

Darouach, M., M. Zasadzinski, \& S. Xu (1994). Full-order observers for linear systems with unknown inputs. IEEE Transactions on Automatic Control 39(3), 606-609.

Ertrac, EPoSS, \& SmartGrids (2012). European Roadmap Electrification of Road Transport 2 Edition. Technical Report June, European Road Transport Research Advisory Council.

Ho, L. M. \& D. Ossmann (2014). Fault Detection and Isolation of Vehicle Dynamics Sensors and Actuators for an Overactuated X-by-Wire Vehicle. pp. 6560-6566.

Isermann, R. (2006). Fault-diagnosis systems: An introduction from fault detection to fault tolerance. Springer.

Jazar, R. N. (2008). Vehicle Dynamics: Theory and Application. Springer.

Rongrong, W. \& W. Junmin (2011). Fault-tolerant control with active fault diagnosis for four-wheel independently-driven electric ground vehicles. In American Control Conference (ACC), 2011, pp. 3954-3959.

Savaresi, S. M. \& M. Tanelli (2010). Active Braking Control Systems Design for Vehicles. Advances in Industrial Control. Springer London.

Ungoren, a. Y., H. Peng, \& H. E. Tseng (2002). Experimental verification of lateral speed estimation methods. Proceedings of the Advanced Vehicle Control (2), 361-366.

Zhang, X., V. Cocquempot, B. Jiang, \& H. Yang (2013). Active fault diagnosis based on fault-tolerant control with control constraints for an electric 4WD vehicle. 10th IEEE International Conference on Control and Automation, 1724-1729.

Table 2: Vehicle Parameter used in the simulations;

\begin{tabular}{|c|c|c|}
\hline Variable & Symbol & Value \\
\hline \hline Vehicle mass & $m$ & $1100 \mathrm{~kg}^{2}$ \\
\hline Yaw inertia & $I_{z}$ & $996 \mathrm{kgm}^{2}$ \\
\hline Distance from front axle and COG & $l_{f}$ & $1.2 \mathrm{~m}$ \\
\hline Distance from rear axle and COG & $l_{r}$ & $1.3 \mathrm{~m}$ \\
\hline Track width & $l_{s}$ & $1.5 \mathrm{~m}$ \\
\hline Aerodynamic drag constant & $K_{w}$ & 0.3401 \\
\hline Wheel radius & $R$ & $0.3 \mathrm{~m}$ \\
\hline Wheel inertia & $I_{w}$ & $1.1 \mathrm{~kg} \mathrm{~m}$ \\
\hline Cornering stiffness front tire & $C_{f}$ & $24500 \mathrm{~N} / \mathrm{rad}$ \\
\hline Cornering stiffness rear tire & $C_{r}$ & $26900 \mathrm{~N} / \mathrm{rad}$ \\
\hline Longitudinal velocity & $V$ & $10 \mathrm{~m} / \mathrm{s}$ \\
\hline
\end{tabular}

